latitudes. The opportunities of observing were not frequent, either from the rarity of the phenomena (which is very possible) or because the dense mass of cloud which is the prevailing feature of those regions prevented their being seen except when exceptionally bright.

Altogether four appearances were noted. The first was 1.30 on the morning of February 9,1874 , in lat. $57^{\circ} \mathrm{S}$. and long. $75^{\circ} \mathrm{E}$., bar. $29^{\circ} \mathrm{O}$ in., ther. $35^{\circ}$. There were brilliant streaks to the westward ; no spectroscopic observations were taken. The second was on February $2 I$ at 9.3O P.M., lat. $64^{\circ}$ S., long. $89^{\circ}$ E., bar. 288 , ther. $31^{\circ}$; one bright white curved streamer extended from Jupiter, which appeared to be near the focus, through Orion and about as far beyond. Under this was what appeared to be a black cloud, but the stars were visible through it. Real cumulus clouds hid great part of the remainder, but there were two vertical flashing rays that moved slowly to the right (west), generally the aurora was still and bright.

On examining the streamer with the spectroscope I found the usual three prominent lines, namely, one yellow-green, one green, the third blue or purple. I looked for the red line but could not find it.

The third aurora was seen on March 3, lat. $53^{\circ} 30^{\prime}$ S., long. $109^{\circ}$ E., bar. $29^{\circ} \mathrm{I}$, ther. $36^{\circ}$, after some days wet and stormy weather. Soon after 8 P.M. the sky began to clear and the moon shone out. Noticing the light to the southward to be particularly bright I applied the spectroscope and found the distinguishing auroral line. About midnight I was called as there were very brilliant auroral clouds. The sky was almost clear, but sonth were two or three brilliant light clouds, colour very white yellow, shape cumulus stratus; from about west to near south extended a long feathery light of the same colour, parallel with the horizon, and between south and west there appeared occasionally brilliant small clouds, the upper edges seemed hairy, and gave one the idea of a bright light behind a cloud. The forms changed, but $I$ did not notice any particular order, perhaps because my attention was particularly directed to examining the light with the spectroscope, and the great cold, for my fingers seemed almost frozen, and the motion of the ship made my task rather difficult. I could trace four lines, three bright, and one rather faint, and by reference to the moon, which was shining brightly, roughly determined their places. They must have been exceedingly bright to show so plainly in full moon. The spectroscope used was one of Grubb's single prism with Iong collimator. A needle point in the eye-piece marked the position of the lines, and a corresponding needle point carried on a frame with the point in the eye-piece and moved by a coarse thread screw, scratched the lines on a plate of blackened glass. I took two plates; - on the first I scratched the auroral lines and the telluric lines visible in the moonlight; on the second I scratched the auroral lines, the telluric lines shown by the moon, and the lines given by carbon in the flame of a spirit lamp; the next morning I verified the lines in sunlight. The lines marked $\mathrm{A}$ are those shown

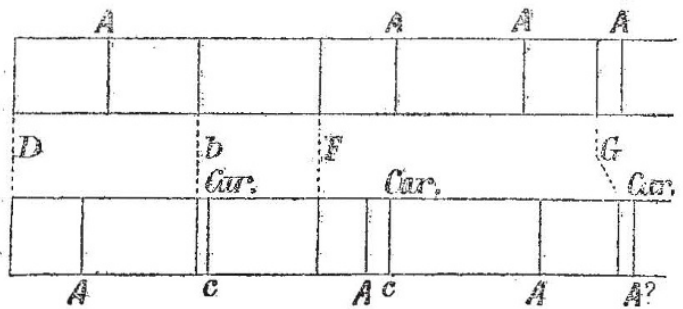

by the aurora, those marked $D, b, F$, and $G$ are the telluric lines, and those marked car. were given by the carbon in the spirit lamp.

The spectrum has been magnified five times from the plates. I cannot account for the different position of the auroral lines in the two plates, as the prism was not moved during the observations that I am aware of.

The fourth aurora was a slight one seen to the southward on March 6 at 8 P.M. It would be worth investigating whether the low barometer has anything to do with the absence of red in the spectrum, the normal state of the barometer is an inch lower in those regions than in more temperate latitudes.

I may as well add that on February 9 the aurora was preceded by a watery sunset, and the day broke aíterwards with high cirrus clouds and clear horizon. On February $2 \mathrm{r}$ the aurora preceded a fine morning, cumulus stratus clouds. On March 3 there was a brilliant sunset followed by a fine morning; and on March 6, after the slight appearance of aurora, the clouds changed to high cirrus.

J. P. Maclear

\section{ABSOLUTE PITCH}

$A \mathrm{~T}$ the present time the question of absolute pitch is attracting attention in consequence of the discrepancy between König's scale and the numbers determined by Appunn's tonometer. This instrument is founded upon the same idea as Scheibler's fork tonometer, and consists of a s.ries of sixty-five harmonium reeds, bridging over an entire octave, and so tuned that each reed gives with its immediate neighbours four beats per second. The application to determine absolute pitch, however, does not require precision of tuning, all that is necessaiy being to count with sufficient accuracy the number of beats per second between each pair of consecutive reeds. The sum of all these numbers gives the difference of frequencies of vibration between the first reed and its octave, which is, of course, the same as the frequency of the first reed itself.

The whole question of musical pitch has recently been discussed with great care by Mr. Ellis, in a paper read before the Society of Arts (May 23, 1877). He finds by original observation with Appunn's instrument $25^{\circ} 4$ as the actual frequency of a König's 256 fork, and Prof. Preyer, of Jena, has arrived at a similar result $(2582)$. On the other hand, Prof. Mayer in America, and Prof. Macleod in this country, using other methods, have obtained numbers not differing materially from König's. The discrepancy is so considerable that it cannot well be attributed to casual errors of experiment ; it seems rather to point to some defect in principle in the method employed. Now it appears to me that there is such a theoretical defect in the reed tonometer, arising from a sensible mutual action of the reeds. The use of the instrument to determine absolute frequencies assumes that the pitch of each reed is the same, whether it be sounding with the reed above, or with the reed below; and the results arrived at would pe vitiated by any mutual influence. In consequence of the ill-understood operation of the wind, it is difficult to predict the character of the mutual influence with certainty; but ("Theory of Sound," $\S \S$ II2-II5) there is reason to think that the sounds would repel one another, so that the frequency of the beats heard when both reeds are sounding, exceeds the difference of the frequencies of the reeds when sounding singly. However this may be, in view of the proximity of consecutive reeds and of the near approach to unison, ${ }^{2}$ the assumption of complete independence could only be justified by actual observation, and this would be a matter of some delicacy. If the mutual influence be uniform over the octave it would require a difference of one beat per minute only to reconcile König's and Appunn's numbers.

As to the amount of the influence I am not in a position to speak with confidence, but I may mention an obser-

I. It must not be forgotten that the vibration of the tongue involves a transference of the centre of inertia, so that there is a direct tendency to set the sounding-board into motion. 
vation which seems to prove that it cannot be left out of account. If two sounds of nearly the same pitch are going on together, slow beats are heard as the result of the superposition of vibrations. Suppose now that a third sound supervenes whose pitch is such that it gives rapid beats with the other two. It is evident that these rapid beats will be subject to a cycle of changes whose frequency is the same as that of the slow beat of the first two sounds. For example, in the case of equal intensities of two sounds there is a moment of silence due to the superposition, of equal and opposite vibrations, and at this moment a third sound would be heard alone and could not give rise to beats. The experiment may be made with tuning-forks, and the period of the cycle will be found to be sensibly the same whether it be determined from the slow beat of the two forks nearly in unizon or from the rattle caused by the simultaneous sounding of a third fork giving from four to ten beats per second with the other two. In the case of forks there is no fear of sensible mutual action, but if it were possible for the third sound to affect the pitch of one of the others the equality of the periods would be disturbed. The observation on Appunn's instrument was as follows :The reeds numbered o and 64 being adjusted to an exact octave, it was found that the beats arising from the simultaneous sounding of reeds 0,63 , and 64 were by no means steady, but passed through a cycle of changes in a period no greater than about five seconds. In order to work with greater certainty a resonator of pitch corresponding in reed 64 was connected with the ear by a flexible tube and adjusted to such a position that the beats between rceds 0 and 64 (when put slightly out of tune) were as distinct as possible, indicating that the gravest tone of reed 64 and the octave over-tone of reed o were of equal intensity. By flattening reed 64 (which can be done very readily by partially cutting off the wind) the beats of the three sounds could be made nearly steady, and then when reed 63 was put out of operation, beats having a 5 seconds' period were heard, indicating that reeds 0 and 64 were in tune no longer. It would appear, therefore, that when reed 63 sounds the pitch of reed 64 is raised, but in interpreting the experiment a difficulty arises from the amount of the disturbance being much in excess of what would be expected from the performance of the instrument when tested in other ways. 1

I come now to an independent determination of absolute pitch, which it is the principal object of the present communication to describe. The method employed may be regarded as new, and it appears to be capable of giving excellent results.

The standard fork, whose frequency was to be measured, is one of König's, and is supposed to execute $128 \mathrm{com}-$ plete vibrations in a second. When placed on its stand (which does not include a resonance box) and excited by a violin bow, it vibrates for a minute with intensity sufficient for the counting of beats. The problem is to compare the frequency of this fork with that of the pendulum of a clock keeping good time. In my experiments two clocks were employed, of which one had a pendulum making about $\mathrm{I} \frac{1}{2}$ complete vibrations per second, and the other a so-called seconds' pendulum, making half a vibration per second. Contrary to expectation, the slower pendulum was found the more convenient in use, and tire numerical results about to be given refer to it alone. The rate of the clock at the time of the experiments was determined by comparison with a watch that

The value of my instrument has been greatly enhanced by the valuable assistance of Mr. Ellis, who was good enough to count the entire series of beats, and to compare the pitch with that of the tuning-forks employed by him in previous investigations. Mr. Ellís, however, is not responsible fo the facts and opinions here expressed. It may be worth mentioning that the steadiness or unsteadiness of the beats heard when three consecutive reeds are sounding simultaneously is a convenient test of the equality of the consecutive intervals. The frequency of the cycle of the four a second beats is equal to the difference of the frequencies of either of the actual both make the intervals exactly equal. was keeping good time, but the difference was found to be too small to be worth considering. In what follows it will be supposed for the sake of simplicity of explanation that the vibrations of the pendulum really occupied two seconds of time exactly.

The remainder of the apparatus consists of an electrically maintained fork interrupter, with adjustable weights, making about $\times 2 \frac{1}{2}$ vibrations per second, and a. dependent fork, whose frequency is about I25. The current from a Grove cell is rendered intermittent by the interrupter, and, as in Helmholtz's vowel experiments, excites the vibrations of the second fork, whose period is as nearly as possible an exact submultiple of its own. When the apparatus is in steady operation, the sound emitted from a resonator associated with the higher fork has a frequency which is determined by that of the interrupter, and not "by that of the higher fork itself; nevertheless, an accurate tuning is necessary in order to obtain vibrations of sufficient intensity. ${ }^{\prime}$ By counting the beats during a minute of time it is easy to compare the higher fork and the standard with the necessary accuracy, and all that remains is to compare the frequencies of the interrupter and of the pendulum. For this purpose the prongs of the interrupter are provided with small plates of tin so arranged as to afford an intermittent view of a small silvered bead carried by the pendulum and suitably illuminated. Under the actual circumstances of the experiment the bright point of light is visible in general in twenty-five positions, which would remain fixed, if the frequency of the interrupter were exactly twenty-five. times that of the pendulum. In accordance, however, with a well-known principle, these twenty-five positions are not easily observed when the pendulum is simply looked at; for the motion then appears to be continuous. The difficulty thence arising is readily evaded by the interposition of a somewhat narrow vertical slit, through which only one of the twenty-five positions is visible. In practice it is not necessary to adjust the slit to any particular position, since a slight departure from exactness in the ratio of frequencies brings all the visible positions into the field of view in turn.

In making an experiment the interrupter is tuned, at first by sliding the weights and afterwards by soft wax, until the interval between successive appearances of the bright spot is sufficiently long to be conveniently observed. With a slow pendulum there is no difficulty in distinguishing in which direction the pendulum is vibrating at the moment when the spot appears on the slit, and it is best to attend only to those appearances which correspond to one direction of the pendulum's motion. This will be best understood by considering the case of a conical pendulum whose motion, really circular, appears to be rectilinear to an eye situated in the plane of motion. The restriction just spoken of then amounts to supposing the hinder half of the circular path to be invisible. On this understanding the interval between successive appearances is the time required by the fork to gain or lose one complete vibration as compared with the pendulum. Whether the difference is a loss or a gain is easily determined in any particular case by observing whether the apparent motion of the spot across the slit (which should have a visible breadth) is in the same or in the opposite direction to that of the pendulum's motion.

In my experiment the interrupter gained one vibration on the clock in about eighty seconds, so that the frequency of the fork was a thousandth part greater than 12.5 or $12.5 \mathrm{I}$. The dependent fork gave the ninth harmonic, with a frequency of $125^{\circ} \mathrm{r}$. The beats between this fork and the standard (whose pitch was the higher) were 180 in sixty seconds, so that the frequency of the standard was as nearly as possible $\mathrm{I} 28^{\circ} \mathrm{I}$, agreeing very closely with König's

I This tuning is effected by prolonging as much as possible the period of the beat heard when the dependent fork starts from rest. This beat may be regarded as due to an interference of the forced and natural notes. 
scale. The error of the determination may amount to ' $I$, but could not, I think, exceed 2 .

I ought to add that the approximate determination of the frequency of the interrupter must be made independently, as the observation on the pendulum does not decide which multiple of $\frac{1}{2}$ nearly coincides with the frequency of the fork. Also the relation between the two auxiliary forks was assumed, and not determined; but as to this there can be no doubt, unless it be supposed that König's scale may be in error to the extent of a whole tone.

RAYLEIGH

\section{A NEW CONDENSING HYGROMETER}

$\triangle \mathrm{NEW}$ apparatus of this kind, invented by $M$. A Alluard, and described by him in La Nature, is distinguished from all those hitherto employed by the two

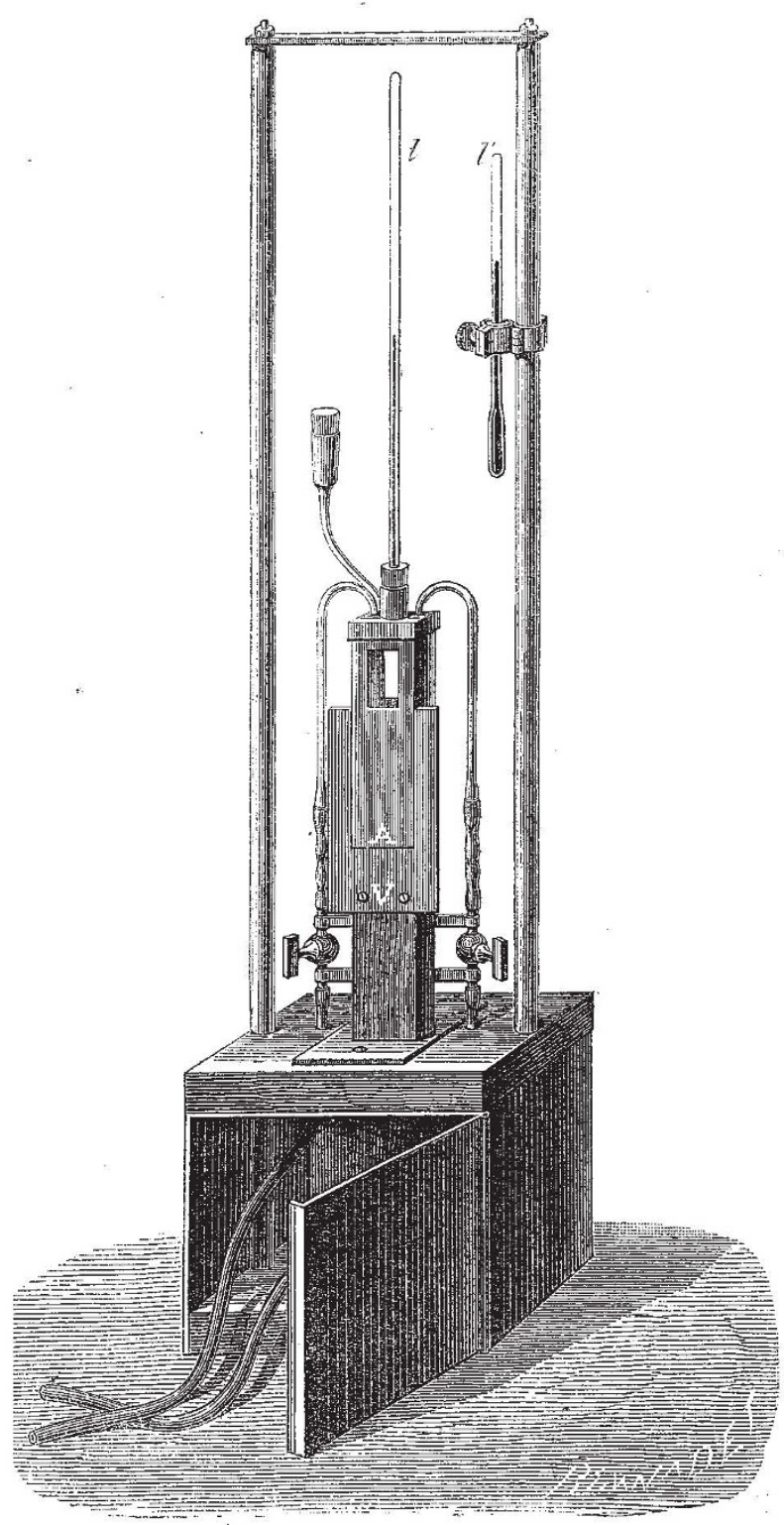

Alluard's Condensing Hygrometer.

following points :-(1) The part on which the deposit of dew is to be observed is a plane well-polished face $A$, of silver or gilt brass; (2) This plane face is set in a plate of silver or brass $\mathrm{v}$, itself gilt and polished, which does not touch it, and which, never being cooled, always preserves its brightness. It results from this arrangement that the deposit of dew is observed with the greatest facility, in such a manner that there is scarcely any difference between the temperatures of the instants when the dew commences and ceases to appear on the instrument properly cooled by the evaporation of ether.

The form of the instrument is that of an upright prism with square base. Its height is eight centimetres and the side of its base eighteen millimetres. Three small copper tubes pass through the upper lid; the first reaches the bottom, and the two others, one surmounted by a funnel for introducing the ether, open only above. 'Two small windows enable us to judge of the agitation of the ether by the aspiration or driving back of the air intended to produce coolness by the evaporation of the volatile liquid ; it is best to work with an aspirator, the aspiration of which we can regulate as we wish. A central tube permits the introduction of a thermometer, $t$, which, placed in the middle of the evaporating liquid, gives the temperature at which the deposit of dew occurs. A small sling thermometer, fixed on the side of a brass support, enables us to determine with precision the temperature of the air whose hygrometric condition we wish to ascertain.

Daniell's condensing hygrometer was formerly modified by M. V. Regnault. He made it an instrument of precision; but his apparatus has not been much used on account of its delicate construction. The deposit of dew, being made on a cylinder of polished silver, is difficult to observe. In the plane face hygrometer of $\mathrm{M}$. Alluard this deposit is very easily seen by contrast, even at some metres distance, especially if care is taken to observe in such a manner as to avoid all reflection from the gilt faces, when they will appar a beautiful ebony black. Its employment being very simple, without losing anything of its precision, there is no reason why it should not come into general use.

Since meteorological observations have multiplied on all sides, the hygrometer has assumed an importance which it had not before. The psychrometer is at present almost exclusively employed. But all physicists know that below zero we cannot trust the results which it gives; it is the same when the air is much disturbed. And yet, almost everywhere, it continues to be employed on these conditions. We hope that the plane face hygrometer, furnished during the winter cold with an aspirator filled with glycerine, will be able to yield accurate results to all who do not fear to devote a few minutes to its working.

\section{OUR ASTRONOMTCAL COLUMN}

EARly OBSERVATIONS OF THE SOlar CORONA.Referring to Mr. Dreyer's letter in Nature (vol. xvi. p. 549), the note in this column relating to the solar eclipse of 1605 was by no means intended to imply that it afforded one of the earliest observations of the corona, nor can the eclipse of Stiklastad, as it hes been usually called, on August 3I, ro3o, be so characterised. Prof. Julius Schmidt, of Athens, had called attention in 1870 to a record of the eclipse of December 22, 968, in Corfu, where he found a reference to the corona, but a much earlier date is assigned by Prof. Grant for the first mention of this phenomenon. It occurs in Philostratus' "Life of Apollonius of Tyana," Book VIII., chap. xxiii., in the

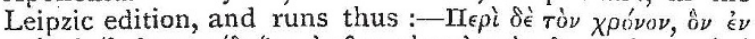

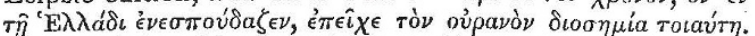

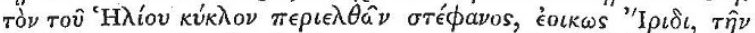

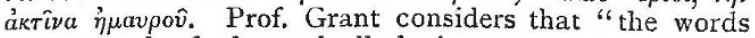
here quoted refer beyond all doubt to a total eclipse of the sun, and that the phenomenon seen encompassing the sun's disc was really as well as verbally, identical with the modern corona." He also points out that Plutarch, 